\title{
Relaxation behavior of aqueous dispersion polysaccharides
}

\author{
Valerii Mank, Oksana Tochkova, \\ Oksana Melnyk, Oleksandr Bessarab
}

\section{National University of Food Technologies, Kyiv, Ukraine}

Keywords:

Polysaccharide

Starch

Dispersion

Relaxation

Structure

Article history:

Received 12.10.2016

Received in revised

form 30.11.2016

Accepted 27.12.2016

\section{Corresponding}

author:

Oksana Tochkova

E-mail:

tochka-ov@i.ua

DOI: $10.24263 / 2304-$

974X-2016-5-4-12

\section{Abstract}

Introduction. Rheological properties of aqueous solutions of polysaccharides showed thixotropic behavior in the destruction of their partially well-maintained structure that occur during physical or thermal effects.

Materials and methods. For experiments prepared 3-15\% dispersion of native potato and corn starches in distilled water. These dispersions kept in an incubator for $30 \mathrm{~min}$ at a temperature $20{ }^{\circ} \mathrm{C}$. The specimens loaded in a cylindrical container reotest at room temperature. After receiving the rheological curve of the sample aqueous dispersion of starch, its left in reotest for 3 hours and then received the rheological curve.

Results and discussion. A new method of relaxation in starch mixtures is that the structural relaxation of polymer units of the polysaccharides in time is slow and long.

According to the literature, only polysaccharides of molecular weight greater than 1 million, for example potato starch, is characterized by the slow relaxation of the structure. Physic-chemical characterization of polysaccharides relaxation, is due to a partially ordered structure with the mutual arrangement of individual chains in a spatial grid.

Under the action of shear stress to reotest destroyed pseudo plastic structure of aqueous dispersions of starch is gradually restored to equilibrium with the system state. The recovery time of the structure is 17 hours.

The slow process of recovery, or structural relaxation of the spatial structure of aqueous dispersions of polysaccharides is due to the adjustment of the spatial grid of the polymer, which is formed due to the existence of transverse chemical bonds.

Similar behavior with a little time structural relaxation typical of other polysaccharides, in particular xanthan, the relaxation time which is about 3 hours.

Conclusion. These characteristics of colloidal systems affect its quality, structural, mechanical and technological properties that must be considered when choosing rational modes of equipment, development of optimal process conditions, the design of automated production control systems. 


\section{Introduction}

Raw materials, semi-finished products and finished products of food processing industries have different structural and mechanical properties such as elasticity, flexibility, strength, creep, thixotropy, relaxation. These properties of food systems depend on temperature, humidity, pressure, mode of transportation, means and timing of storage. These factors affect the behavior of the structure during its deformation when machining: grinding, forming, stamping and others $[1,2,12,13]$.

To improve the organoleptic, structural, mechanical, physical and chemical characteristics of food products used polysaccharides. They provide some form of products such as sauces, puddings, confectionery, comminuted meat, fish products, and low fat dairy products. In a food system, the roles of polysaccharides are to stabilize the structure and interact with other components to deliver or maintain nutrient and flavor. For example, polysaccharides can serve as thickening agents for sauces, cream soups, and pie fillings; colloidal stabilizers for salad dressing; moisture retention for cake toppings; gel-forming agents for gum confections; binders for wafers and ice cream cones; and coating and glazing agents for nut meats and candies.

Among natural polysaccharides starch occupies a unique position. It is has a wide range of applications in many industries and technology. This is what causes the large number of studies state starches of different botanical origin in conditions the action of various factors that appear in the literatures recently.

Natural polysaccharides, unlike synthetic, are characterized by a partially order structure which is formed in the process of synthesis and growth. This feature of biological macromolecules polysaccharides is due, primarily, their chiral structure, paired with the existence of hydrogen bonds included water molecules in their structure [1].

As you know, there is change in the nature of intermolecular inter actions in biomacromolecules starch in a temperature range of $40-70{ }^{\circ} \mathrm{C}$ and converts it ordered structure $[1,2]$. This is also confirmed by experiments in the dehydration process gelatinized starch [3].

The structural features of starch reflected in the rheological behavior of its aqueous dispersions. During processing, starch dispersions will be subjected to combined high heating and shearing that affect their rheological change as well as the final characteristics of the product. Starch gelatinization, especially granular swelling, changes the rheolgical properties of aqueous dispersions. The subsequent retrogradation will further modify the rheolgical properties of dispersions. Depending on the starch source and concentration, the final structure of starchy products will give a thickened solution or a gelled structure. Thus, the rheological curves of starch dispersions are characteristic of non-Newtonian fluid while at low shear stresses dispersions behave as elastoplastic system. Increasing the tension is the destruction structural formations and the system behaves as a classical fluid. These properties of the system due to the presence of intermolecular interactions and chaotic thermal motion of molecules [4-6].

It is suggested that after the removal of the applied stress to the system is slow restoration of the original structure, i.e. relaxation. Relaxation period is characterized by the speed system transition from a non equilibrium thermodynamic condition caused by external influence, in the state of thermodynamic equilibrium.

To confirm is assumption studied the rheological behavior of starch aqueous dispersions after a certain period of time after receiving another rheological curve. 


\section{Materials and methods}

Materials. For experiments prepared 3-15\% dispersion of native potato and corn starches in distilled water.

Method. These dispersions kept in an incubator for $30 \mathrm{~min}$ at a temperature $20^{\circ} \mathrm{C}$. The specimens loaded in a cylindrical container reotest at room temperature. After receiving the rheological curve of the sample aqueous dispersion of starch, its left in reotest [14] for 3 hours and then received the rheological curve $[1,2,4]$.

\section{Results and discussion}

Rheolgical curves with time become more nonlinear and approaching in shape to the original curve (Figure 1, curve 1). This indicates that the destroyed under shear stress resistor pseudo plastic structure aqueous dispersions of starch gradually restored to equilibrium at the given conditions of the system.

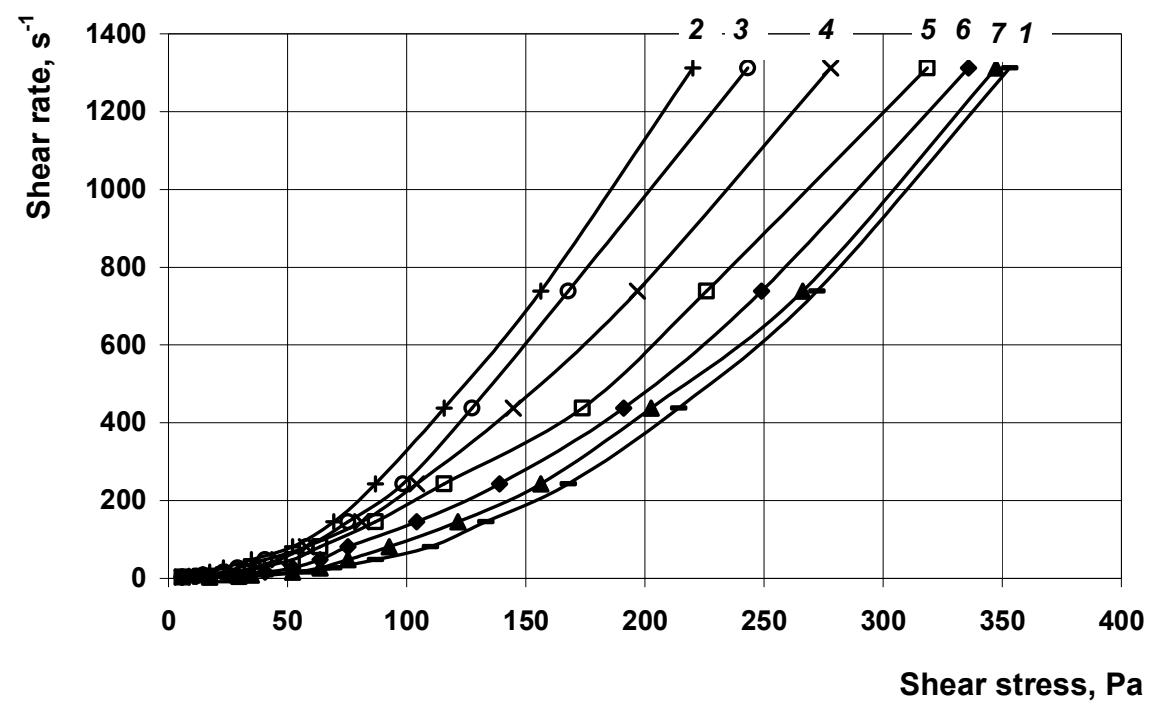

Figure 1. Rheograms for $5 \%$ dispersion of potato starch, heat treated at $20^{\circ} \mathrm{C}(1)$ taken every 3 hours (2-7)

In general, the deformation that occurs as a result of shear stresses consists of elastic and viscous components. Elastic component are on Hooke's law [4, 7]:

$$
\left(\frac{d \gamma}{d \tau}\right) e l=\frac{d P}{(G d \tau)}
$$

where $P$ - shear stress, $G$ - modulus of elasticity. 
Any structured system described by Hooke's law only to a certain limit, above which comes the new state of deformation.

Viscous component is determined from the equation Newton [4, 7]:

$$
\left(\frac{d \gamma}{d \tau}\right) v=\left(\frac{P}{\eta}\right)
$$

where $\eta$ - viscosity.

Summing up two components obtained the Maxwell equation [4, 7]:

$$
\frac{d y}{d \tau}=\frac{d P}{\left(G d \tau+\frac{P}{\eta}\right)}
$$

As is known [4, 5, 7], unauthorized restoration structure of aqueous dispersions of various substances occurs exponentially to confirm this model, analyzed the rheological behavior of starch aqueous dispersions at regular intervals after the complete destruction of their structure. It was assumed that the value of shear rate that is fixed for each intermediate rheolgical curve in Figure 1 at shear stress of $200 \mathrm{~Pa}$, also varies exponentially depending on the recovery time structure.

The dependence of shear rate of starch structure, $v$ on time at fixed shear stress $200 \mathrm{~Pa}$ can be represented by the following expression [7]:

$$
\gamma=\gamma_{0} e^{-\frac{\tau}{\tau_{p}}}
$$

where $\gamma$ and $\gamma_{0}$ are shear rate $\mathrm{s}^{-1}$, for observation $\tau$ and relaxation time, $\tau_{p}$.

Studies to determine the $5 \%$ dispersion of potato starch presented on Figure 2.

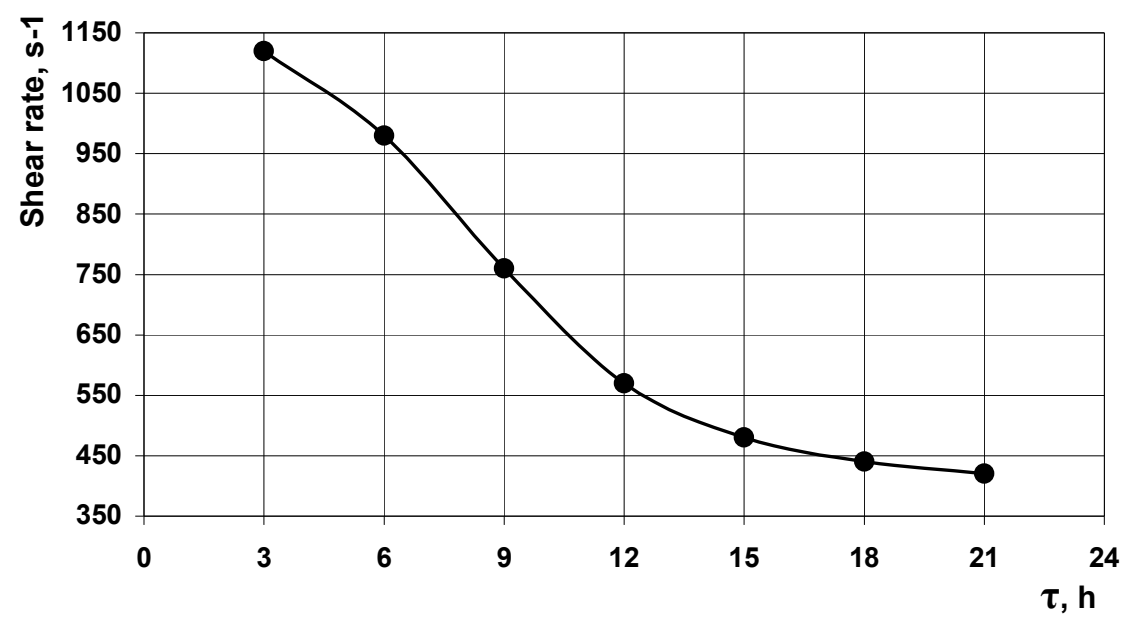

Figure 2. The dependence of shear rate on time at fixed shear stress $(200 \mathrm{~Pa})$ for $5 \%$ dispersion of potato starch, heat treated at $20^{\circ} \mathrm{C}$ 
To determine the period of relaxation structure constructed dependence $\ell n \gamma-\tau$ (Figure 3).

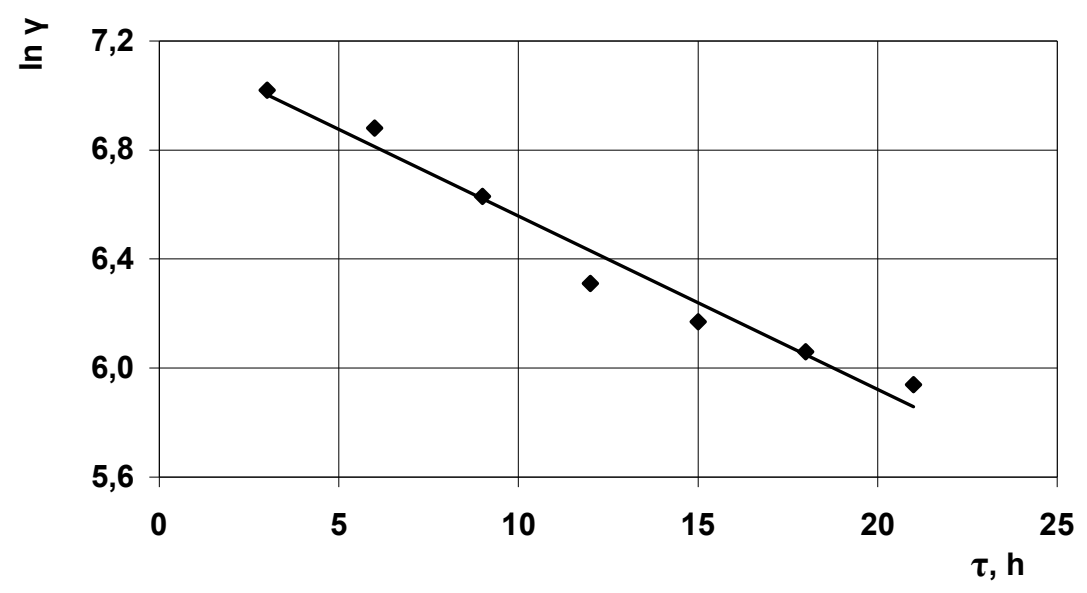

Figure 3. The dependence of shear rate on time at fixed shear stress

Relaxation period was calculated as $\operatorname{tg}=\frac{1}{\tau_{p}}$ the slope angle of the line $\ln \gamma=f \tau$. The relaxation period is 17 hours.

Linear dependence of the logarithm of shear speed from time observed the restoration of equilibrium in the system shown in Figure 4. It confirms the proposed mechanism of restoring equilibrium in the system after mechanical excitation. The rate of structure relaxation decreases evaluates from equation (1) is 17 hours, which indicates very slow process of restoring equilibrium of the sequence moving individual units of the polymer in a more convenient location moving individual units of the polymer in a more convenient location.

It is natural to assume that the recovery rate of equilibrium will depend on the complexity of the structure of the polymer. This is confirmed by similar experiments with simpler polysaccharides with a molecular weight of less than 1000000 , while the molecular weight of starch 1000 000. As an example, in Figure 4 shows the experimental determination of relaxation in aqueous solutions of the xanthenes gum. Time structural relaxation of the xanthenes gum is 210 minutes (Figure 4).

Very slow relaxation process spatial patterns are characteristic of cross-linked polymers in highly elastic state where the relaxation process is due to the restructuring of the spatial networks formed by intermolecular chemical bonds.

In the studied of polysaccharides aqueous dispersions maybe similar relaxation process that caused flexible friction a large areas of polymer chains. 


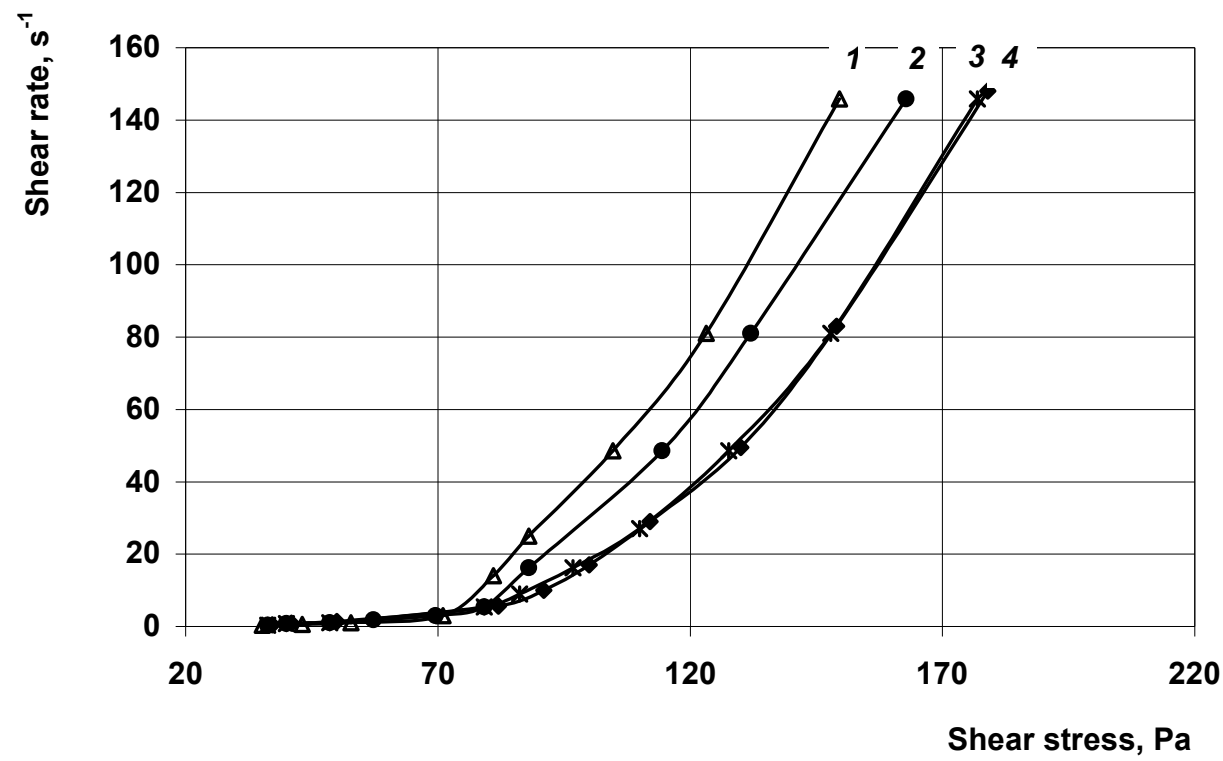

Figure 4. Rheograms for $5 \%$ dispersion of xanthenes, heat treated at $70^{\circ} \mathrm{C}$ (1) taken every 3 hours (2-4)

\section{Conclusions}

Study rheology behavior of aqueous starch dispersions in various stages of restoration non-Newtonian liquids structure and consideration possible model of their relaxation have confirmed the existence of ordered structures formed by interaction with water molecules surrounding hydrocarbon chains of biopolymers.

Under the influence of shear stresses in rheostat, the structural elements in starch mixtures are destroyed. Dispersion system became into a state of Newtonian liquid. Rebalancing to the original state in the secomplex systems is slow enough.

Developed method of changing the times of the structural relaxation of the carbohydrate chains of the polymer.

This behavior aqueous dispersions of polysaccharides must be considered when considering the in termolecular inter actions and structure of polymer solutions, as well as the development of technology using polysaccharides as food additives.

\section{References}

1. Mank V., Melnyk O., Bakhmach V. (2014), Anomalous properties in agueous solutions of polysacchariedes, Ukrainian Journal of Food Science, 2 (2), pp. 236-243.

2. Bertolini C.A. (2010), Starches: characterization, properties, and applications, Taylor and Francis Group, New York. 
3. Xiaowen L., Wu L., Wang J., Li J., Qin Y. (2011), Characterization of Water Binding and Dehidration in Gelatinized Starch. J.Agric.Food Chem., 59(1), pp. 256-262.

4. Malkin A. J., Askadskyi A.A., Kovryga V.V. (1978), Metody izmereniya mekhanicheskykh svoistv polymerov, Khimiya, Moscow.

5. Gorbatov A.V., Machykhyn S.A., Maslov A. M., (1982), Strukturno-mechanicheskie kharakterystyky pischevukh produktov, Lehkaya i pischevaya promyshlenost, Moscow

6. Xue T., Yu L., Xie F., (2008), Rheological properties and phase transition of starch under shear stress, Food Hydrocolloids, 22, pp. 973-978.

7. Padokhyn V.A., Kokina N.P., (2007), Fiziko-mekhanicheskie svoistva suria $i$ pischevukh produktov, Ivanovo.

8. Flad E. (1999), Interphase boundary gas-solid, Moscow, pp. 129-147.

9. Olga Rybak (2013), Some aspects of the formation of emulsions and foams in food industry, Ukrainian Journal of Food Science, 1(1), pp. 41-49.

10. Akselrud G.A., Articular M.A. (1993), Introduction to capilano-chemical technology, Moscow.

11. Schrader M. E., Yariv S. (1990), Wettability of Clay Minerals, Ibid, 136(1), pp. 85-94.

12. Lugovska O., Sydor V. (2014), Effect of starch as hydrocolloids for formation of a stable emulsion system in food, Ukrainian food journal, 3(2), pp. 202-210.

13. Ai Y., Jane J.1. (2016), Starch: Structure, Property, and Determination, Encyclopedia of Food and Health, Academic Press, pp. 165-174.

14. Natalia Dacka, Olena Podobii (2014), The sour milk drink with antioxidant properties, Ukrainian Journal of Food Science, 2(2), pp. 205-212. 